\title{
Quantitative structure-activity relationships (QSARs) using the novel marine algal toxicity data of phenols
}

\author{
M. Doğa Ertürk ${ }^{\mathrm{a}}$, Melek Türker Saçan ${ }^{\mathrm{a}, *}$, Marjana Novic $^{\mathrm{b}}$, Nikola Minovski $^{\mathrm{b}}$ \\ a Boğaziçi University, Institute of Environmental Sciences, 34342, Hisar Campus, Bebek, Istanbul, Turkey \\ ${ }^{\mathrm{b}}$ National Institute of Chemistry, Hajdrihova 19, 1000 Ljubljana, Slovenia
}

\section{A R T I C L E I N F O}

\section{Article history:}

Accepted 13 June 2012

Available online 29 June 2012

\section{Keywords:}

Marine alga

Toxicity

Phenols

QSAR

MLR

CPANN

\begin{abstract}
A B S T R A C T
The present study reports for the first time in its entirety the toxicity of 30 phenolic compounds to marine alga Dunaliella tertiolecta. Toxicity of polar narcotics and respiratory uncouplers was strongly correlated to hydrophobicity as described by the logarithm of the octanol/water partition coefficient (Log P). Compounds expected to act by more reactive mechanisms, particularly hydroquinones, were shown to have toxicity in excess of that predicted by Log P. A quality quantitative structure-activity relationship (QSAR) was obtained with Log P and a 2D autocorrelation descriptor weighted by atomic polarizability (MATS3p) only after the removal of hydroquinones from the data set. In an attempt to model the whole data set including hydroquinones, 3D descriptors were included in the modeling process and three quality QSARs were developed using multiple linear regression (MLR). One of the most significant results of the present study was the superior performance of the consensus MLR model, obtained by averaging the predictions from each individual linear model, which provided excellent prediction accuracy for the test set $\left(Q_{\text {test }}^{2}=0.94\right)$. The four-parameter Counter Propagation Artificial Neural Network (CP ANN) model, which was constructed using four out of six descriptors that appeared in the linear models, also provided an excellent external predictivity $\left(Q_{\text {test }}^{2}=0.93\right)$.

The proposed algal QSARs were further tested in their predictivity using an external set comprising toxicity data of 44 chemicals on freshwater alga Pseudokirchneriella subcapitata. The two-parameter global model employing a 3D descriptor (Mor24m) and a charge-related descriptor $\left(C_{\text {ortho }}\right)$ not only had high external predictivity $\left(Q_{e x t}^{2}=0.74\right)$, but it also had excellent external data set coverage (\%97).
\end{abstract}

(C) 2012 Elsevier Inc. All rights reserved.

\section{Introduction}

Phenols have been in production since 1860s [1]. They have been used as intermediates in the synthesis of dyes, pigments, phenolic resins, pesticides and herbicides as well as in many industries and consumer products $[2,3]$. Owing to their ubiquitous use for domestic, agricultural and industrial purposes they have become widely distributed in nature $[3,4]$. Due to their persistence in the environment and eliciting adverse effects on living organisms, there has been an interest in determining their potential hazards to various organisms including bacteria, daphnia, protozoa and fish using standardized bioassays [5].

From a regulatory perspective, information regarding toxicity is required for various trophic levels to properly evaluate the possible risks that chemicals may pose to the environment. In this context, algal toxicity data is needed to carry out a proper risk

\footnotetext{
* Corresponding author. Tel.: +90 21235945 98; fax: +90 2122575033 .

E-mail address: msacan@boun.edu.tr (M.T. Saçan).
}

assessment. From an ecological perspective, algae form the base of food webs (i.e. primary producers); they provide food for higher trophic levels and produce oxygen which is vital for the sustainability of life in aquatic environments. Apart from the crucial role they play in the ecosystems, their ubiquitous distribution throughout the globe, ease of collection and culturing, and rapid growth rate make them ideal for laboratory testing [6]. However, as indicated by Cronin et al. [7], while there are relatively large toxicity databases for the fish and crustacean, which represent higher trophic levels, limited toxicity data are available for the primary producers.

The lack of marine algal toxicity data is even more pronounced. The reasons behind the lack of marine ecotoxicity data in general has been attributed to the fact that urbanization has rendered freshwater ecosystems more vulnerable to the chemical releases which in turn made the protection of freshwater communities the first priority by regulatory schemes. Consequently, there is substantial amount of information available on the toxicity of chemicals to freshwater organisms while there are relatively fewer data on the effect of chemicals to marine organisms, in particular 
to aquatic plants and algae [8]. The insufficient marine algal toxicity data has been highlighted in various studies that aimed to exploit relatively abundant freshwater ecotoxicity data in lieu of marine data $[9,10]$. The workshop organized by the European Centre for Ecotoxicology and Toxicology of Chemicals on the probabilistic approaches for marine hazard assessment [11] once again stressed the need for marine algal toxicity data to be able to fill the data gap present in marine ecotoxicity. However, the data gap in this domain of ecotoxicology is almost impossible to fill with exhaustive testing of each chemical. As the experimental determination of toxicity is a costly and time consuming process, quantitative structure-activity relationship (QSAR) studies can provide a useful tool for predicting the toxicities of untested chemicals.

To be able to exploit the merits of QSARs in marine ecotoxicity, the first task is to generate reliable data that can be used in model development. In this study, the toxicity of 30 phenolic compounds, including phenol, chlorophenols and polyphenols, to the marine alga Dunaliella tertiolecta, which is regarded as a species representative of marine environments [6] and a recommended ecotoxicity test species in standard methods [12], is presented for the first time in its entirety. The marine algal toxicity data was determined according to the standardized procedures for algal growth inhibition tests $[12,13]$ in the same laboratory, by the same researcher; therefore, should meet the requirements for quality data. Based on the classification of phenols according to their mode of action (MOA) against the protozoan Tetrahymena pyriformis [14], the marine algal data set can be expected to include polar narcotics $(n=19)$, respiratory uncouplers $(n=4)$ and reactive chemicals (proelectrophiles, pro-redox cyclers, $n=7$ ). Considering the fact that approximately $70 \%$ of industrial organic pollutants exhibit a narcotic mode of toxic action [15], and the presence of compounds having different MOAs, dataset presented in this study can be seen as a miniaturized model of industrial chemical space; as such it provides a suitable basis upon which to explore the development of marine algal QSARs.

The OECD principles [16] require that models should have (a) a defined endpoint; (b) an unambiguous algorithm; (c) a defined domain of applicability; (d) appropriate measures of goodness-of-fit, robustness and predictivity; and (e) a mechanistic interpretation, if possible. In the guidance of these principles, several QSAR models were developed using multiple linear regression (MLR) without taking the MOA of each chemical into account (i.e. global QSARs). For model development, a large descriptor pool comprising 2D and 3D descriptors as well as the logarithms of the octanol/water partition coefficient (Log P) was used. A separate descriptor pool excluding 3D descriptors was created to be able to construct QSARs employing only 2D descriptors and Log P in an attempt to develop and evaluate the performance of marine algal QSARs with and without 3D descriptors.

Several validation criteria were applied to the developed models to ensure that models were robust, predictive and not chance correlations. A consensus MLR model, which was formed by averaging the predicted toxicity for every compound using the proposed MLR models, was also tested in its predictive ability. In an attempt to better understand and interpret the linear models, whole data was decomposed into several subsets based on structural and mechanistic similarity of phenols. Considering the non-linear nature of toxicity, an attempt was also made to develop a Counter Propagation Artificial Neural Networks (CP ANN) model using the significant descriptors that appeared in the linear models. Finally, the proposed QSARs were tested in their predictivity using an external dataset collated by Furusjö et al. [17], comprising the 96-h growth inhibition data of 45 compounds towards freshwater alga Pseudokirschneriella subcapitata (also known as Selenastrum capricornutum).

\section{Materials and methods}

\subsection{Test chemicals, D. tertiolecta growth inhibition test, external data set}

A total of 30 hydroxylated aromatics were selected for toxicological assessment. The chemicals used in this study were purchased from Sigma-Aldrich, Germany. Phenol was obtained from Merck, Germany. The majority of the chemicals had purity $\geq 98 \%$. No further purification was undertaken.

Toxicity data $\left[\log _{10}\left(\mathrm{IC}_{50}{ }^{-1}\right)\right.$, in $\mathrm{mmol} / \mathrm{L}$, denoted as $\left.\mathrm{pT}\right]$ were determined in algal growth inhibition assays utilizing the unicellular marine alga $D$. tertiolecta. The toxicity data of phenol and 13 chlorophenols was published previously [18] and the rest is presented for the first time in the present study. Assays were conducted according to the standardized test protocols $[12,13]$. The inhibitory concentrations of the test substance that inhibits algal growth by $50 \%\left(\mathrm{IC}_{50}\right)$ at the end of $48 \mathrm{~h}, 72 \mathrm{~h}$ and $96 \mathrm{~h}$ to $D$. tertiolecta were determined. The details about the bioassays can be found elsewhere [18].

The external data set comprising the 96-h growth inhibition data $\left(p \mathrm{EC}_{50}\right.$, in $\left.\mathrm{mmol} / \mathrm{L}\right)$ of 45 compounds towards freshwater alga P. subcapitata was obtained from Furusjö et al. [17]. One chemical in this data set, namely octafonium chloride is a salt; therefore, this molecule was excluded from analysis. Consequently, the external prediction was carried out using 44 compounds. The data set comprises phenolic compounds including catechols, guaiacols and chlorophenols as well as aromatic compounds which are structurally different than phenols such as aniline, nitrofurazone, morpholine, acridine, 3-amino-1,2,4-triazole, furaltadone, tebuthiuron, Fennosan F50, chlorobenzene(s) and also straightchained alkyl amines (e.g. dibutyl amine, diethyl amine, diethyl nitrosamine, etc.).

\subsection{Molecular descriptors and model development}

Log P values were extracted from EPI Suite (version 4.1, 2011, United States Environmental Protection Agency). The other molecular descriptors used in the modeling process were calculated using two software packages, namely Dragon (version 5.4., 2006, Talete, Milano, Italy) and CODESSA Pro (2002, CUniversity of Florida, Gainesville, USA). Prior to the calculation of descriptors, geometry optimization of the molecules was carried out in Spartan (version 06, 2006, Wavefunction, Inc., Irvine, CA, USA) using semi-empirical PM3 method. The geometry optimized molecules were then used to calculate the descriptors.

1664 and 818 molecular descriptors were originally retrieved from the Dragon 5.4 and CODESSA Pro software, respectively. Constant descriptors were excluded to minimize the redundant information and the reduced set of 1762 molecular descriptors were imported to CODESSA (version 2.2, 1996, CUniversity of Florida, Gainesville, USA) using an in-house developed software application [19]. All descriptors were analyzed using the CODESSA 2.2 software with heuristic option, which is a suitable option for descriptor selection [20]. A separate descriptor pool excluding 3D descriptors was used to construct QSARs employing only 2D descriptors and Log P.

The heuristic search was restricted to three variables per model to be able to develop simple and informative linear QSARs. The obtained models at the end of heuristic method were then investigated for a possible co-linearity among the descriptors as judged by the Variance Inflation Factor (VIF) in SPSS (version 18, 2009, SPSS, Inc., Chicago, IL, USA). The global models that failed to predict the toxicity of a compound within 2.5 standardized residuals was not qualified for reporting. The acceptable MLR models were then used to form a consensus MLR model by averaging the predicted toxicity 
for every compound. CP ANN modeling was carried out using the modules developed at the Slovenian National Institute of Chemistry [21].

\subsection{Model validation}

In order to obtain compounds for the test set which was used for external validation of the proposed models, the available experimental data for 30 phenols was split into training and test set (used for external validation) prior to descriptor selection as follows: the compounds were ordered according to their descending toxicity values. Out of 30 compounds, 6 clusters were formed which consist of 5 compounds. The 3rd compound in each cluster was assigned to the test set. As a result, training and test sets were formed with 24 and 6 compounds, corresponding to $80 \%$ and $20 \%$ of the dataset, respectively. The internal validation of the models was verified by leave-one-out $\left(r_{L O O}^{2}\right)$ and leave-two-out $\left(r_{L 2 O}^{2}\right)$ cross-validation. The QSARs were constructed using the training set compounds and the external predictivity of these models was estimated on the predicted values in the test sets by $Q_{\text {test }}^{2}$ (or $Q_{\text {ext }}^{2}$ ) according to the following equation [22]:

$Q_{\text {test }}^{2}=1-\frac{\left[\sum_{i=1}^{n_{\text {test }}}\left(y_{i}-\hat{y}_{i}\right)^{2}\right] / n_{\text {test }}}{\left[\sum_{i=1}^{n_{\text {training }}}\left(y_{i}-\mu_{\text {tr }}\right)^{2}\right] / n_{\text {training }}}$

where $y_{i}$ and $\hat{y}_{i}$ are, the observed and predicted (over the test set) values of the dependent variable, respectively; $\mu_{t r}$ is the mean value of the dependent variable for the training set. Although models with $r_{L O O}^{2}$ and $Q_{\text {test }}^{2}$ values higher than 0.5 are considered acceptable in terms of a model's predictivity [23], we reported those higher than 0.7 to avoid a list of models that could be confusing. The models were selected based on the coefficient of determination $\left(r^{2}\right)$, the leave-one-out cross-validation $\left(r_{L O O}^{2}\right)$, the leave-two-out crossvalidation $\left(r_{L 2 O}^{2}\right)$ and the root mean square error for the training set (RMSEt). After the selection of the models, their external validation was assessed for the test set using $Q_{\text {test }}^{2}$ (Eq. (1)) and root mean square error for the test set (RMSEp). The performance of the proposed QSARs in predicting the toxicity of chemicals in the external set obtained from Furusjö et al. [17] was also assessed by $Q_{\text {ext }}^{2}$ (Eq. (1)) and the root mean square error of the external set $\left(R M S E_{\text {ext }}\right)$.

Additionally, the following criteria [24] were applied to the test and external sets to further assess the predictive power of the proposed models:

$\frac{R^{2}-R_{0}^{2}}{R^{2}}<0.1$ and $0.85 \leq k \leq 1.15$

or

$\frac{R^{2}-R_{0}^{\prime 2}}{R^{2}}<0.1$ and $0.85 \leq k^{\prime} \leq 1.15$

and

$\left|R_{0}^{2}-R_{0}^{\prime 2}\right| \leq 0.3$

where $R^{2}$ is the squared correlation coefficient between observed and predicted values for the test set $(n=6) ; R_{0}^{2}$ and $k$ are the correlation coefficient and slopes of the linear regression between the observed and predicted values when intercept was set to zero. The predicted versus observed and observed versus predicted correlation coefficients and slopes are different and therefore the latter were designated as $R_{0}^{\prime 2}$ and $k^{\prime}$, respectively.

Finally, the QSARs were subjected to $Y$-randomization as executed in Molegro Data Modeler (version 2.6.0, 2011, Molegro ApS, Aarhus, Denmark) to ensure that the developed relationships are not chance correlations. In this technique, only the dependent variable is randomly re-ordered while the independent variables are left untouched and a new fit $\left(r_{r}^{2}\right)$ is obtained for the distorted relationship. In this study, this procedure was repeated 30 times for each proposed QSAR and the mean $r_{r}^{2}$ was reported for each model. In this so-called model randomization, the resulting models are expected to have lower squared correlation coefficient $\left(r_{r}^{2}\right)$ compared to the original relationship $\left(r^{2}\right)$ since the link between the structure and activity is severed [25]. For the evaluation of the randomization, we have used $R_{p}^{2}$ in this study as proposed by Roy et al. [25] which is calculated by the following formula:

$R_{p}^{2}=r^{2} *\left(r^{2}-r_{r}^{2}\right)^{1 / 2}$

The $R_{p}{ }^{2}$ should be greater than 0.5 to ensure that model was not obtained by chance.

\subsection{Applicability domain}

Any given QSAR should make reliable predictions within its applicability domain (AD) for untested chemicals. In the present study, the chemical domain of the phenols was investigated by the leverage approach to verify the prediction reliability. To visualize the AD of QSAR models, the standardized residuals were plotted against leverages. The obtained graphical representation was used to detect both the response outliers (Y outliers) and the structurally influential chemicals (X outliers) in the models. In this plot, the horizontal and vertical lines delineate the limits of acceptable values; the former for the Y outliers (i.e. compounds with standardized residuals greater than 2.5 standard deviation units) and the latter for $X$ outliers, respectively. The limit of X outliers is determined by their warning hat values $\left(h^{*}\right)$ calculated by $3 p^{\prime} \mid n$, where $p^{\prime}$ is the number of model variables plus one, and $n$ is the number of compounds in the model [26]. Zhu et al. [27] stated that only a certain fraction of compounds in any external data set is expected to fall within a model's AD and this fraction is referred to as the data set coverage. Therefore, the proposed QSARs were also evaluated in terms of their coverage of the compounds in the external data set (\% coverage).

\section{Results and discussion}

Toxicity data to $D$. tertiolecta for a total of 30 phenolic compounds are presented in Table 1 together with the expected MOA, interaction type and descriptor values for each compound.

For all exposure durations, the least toxic compound was found to be resorcinol while the most toxic compound was pentachlorophenol. The marine algal toxicity of phenols determined at the end of 48-h, 72-h and 96-h had excellent correlation among each other (results not shown). It was observed that phenols tended to be slightly more toxic at 48-h exposure; therefore, we used the 48-h toxicity data as the dependent variable to be conservative in the modeling process. The toxicity values for this exposure duration varied uniformly over approximately 3.5 fold range (from -0.42 to 3.03 on a logarithmic scale).

The relationship between marine algal toxicity $\left(p \mathrm{~T}_{48 \mathrm{~h}}\right)$ and hydrophobicity as described by Log $\mathrm{P}$ is illustrated in Fig. 1 . The CAS numbers, Log P values and marine algal toxicity of the tested phenols at the end of 48-h, 72-h and 96-h exposures can be found in the supplementary materials (Table S1).

As expected, there is a strong trend between hydrophobicity and toxicity for polar narcotics and respiratory uncouplers (collectively referred to as compounds acting through non-covalent mediated mechanisms, Table 1). On the other hand, compounds expected to act by more reactive mechanisms were shown to have toxicity in excess of that predicted by hydrophobicity (Fig. 1). This 
Table 1

Mode of action (MOA), interaction type, marine algal toxicity and descriptors used in the models.

\begin{tabular}{|c|c|c|c|c|c|c|c|c|c|c|c|c|}
\hline ID & Compound & $\mathrm{MOA}^{\mathrm{a}}$ & Interaction type ${ }^{c}$ & $p \mathrm{~T}_{48}$ & $p \mathrm{~T}_{72}$ & $p \mathrm{~T}_{96}$ & Mor24m & $C_{\text {ortho }}$ & Mor24p & HATS7e & Mor18u & $\left(k_{1} \max ^{\mathrm{a}}\right)$ \\
\hline \multicolumn{13}{|c|}{ Training set } \\
\hline 1 & $\mathrm{P}$ & Polar narcotic & Non-covalent & $-0.24( \pm 0.03)^{\mathrm{d}}$ & $-0.28( \pm 0.03)$ & $-0.30( \pm 0.03)$ & 0.068 & -0.144 & 0.046 & 0 & -0.50 & 9.08 \\
\hline 2 & $2-\mathrm{CP}$ & Polar narcotic & Non-covalent & $0.44( \pm 0.04)$ & $0.34( \pm 0.02)$ & $0.31( \pm 0.01)$ & 0.031 & -0.131 & 0.043 & 0 & -0.43 & 9.14 \\
\hline 4 & $4-\mathrm{CP}$ & Polar narcotic & Non-covalent & $0.81( \pm 0.03)$ & $0.67( \pm 0.04)$ & $0.63( \pm 0.02)$ & 0.107 & -0.166 & 0.061 & 0 & -0.47 & 9.05 \\
\hline 5 & 2,3-DCP & Polar narcotic & Non-covalent & $0.98( \pm 0.04)$ & $0.92( \pm 0.01)$ & $0.88( \pm 0.01)$ & 0.170 & -0.135 & 0.070 & 0 & -0.37 & 9.12 \\
\hline 6 & 2,4-DCP & Polar narcotic & Non-covalent & $1.23( \pm 0.04)$ & $1.20( \pm 0.02)$ & $1.14( \pm 0.02)$ & 0.103 & -0.155 & 0.067 & 0 & -0.40 & 9.10 \\
\hline 7 & $2,5-\mathrm{DCP}$ & Polar narcotic & Non-covalent & $0.98( \pm 0.01)$ & $0.97( \pm 0.02)$ & $0.95( \pm 0.01)$ & 0.042 & -0.133 & 0.066 & 0 & -0.41 & 9.13 \\
\hline 8 & 2,6-DCP & Polar narcotic & Non-covalent & $0.43( \pm 0.03)$ & $0.30( \pm 0.03)$ & $0.25( \pm 0.02)$ & 0.004 & -0.121 & 0.051 & 0 & -0.44 & 9.17 \\
\hline 10 & 3,5-DCP & Polar narcotic & Non-covalent & $1.71( \pm 0.05)$ & $1.46( \pm 0.03)$ & $1.39( \pm 0.03)$ & 0.155 & -0.151 & 0.108 & 0 & -0.42 & 9.06 \\
\hline 11 & 2,3,4-ТCP & Polar narcotic & Non-covalent & $1.88( \pm 0.04)$ & $1.68( \pm 0.03)$ & $1.65( \pm 0.04)$ & 0.329 & -0.167 & 0.095 & 0 & -0.31 & 9.08 \\
\hline 12 & $2,3,5-\mathrm{TCP}$ & Polar narcotic & Non-covalent & $1.78( \pm 0.04)$ & $1.78( \pm 0.04)$ & $1.69( \pm 0.01)$ & 0.209 & -0.139 & 0.103 & 0 & -0.35 & 9.11 \\
\hline 13 & 2,3,6-ТCP & Polar narcotic & Non-covalent & $0.80( \pm 0.01)$ & $0.60( \pm 0.02)$ & $0.58( \pm 0.02)$ & 0.144 & -0.125 & 0.074 & 0 & -0.39 & 9.15 \\
\hline 15 & 2,4,6-ТCP & Polar narcotic & Non-covalent & $0.75( \pm 0.02)$ & $0.68( \pm 0.01)$ & $0.67( \pm 0.01)$ & 0.099 & -0.147 & 0.081 & 0 & -0.42 & 9.12 \\
\hline 16 & $3,4,5-\mathrm{TCP}$ & Polar narcotic ${ }^{\mathrm{b}}$ & Non-covalent & $2.22( \pm 0.02)$ & $2.15( \pm 0.03)$ & $2.15( \pm 0.03)$ & 0.373 & -0.189 & 0.123 & 0 & -0.33 & 9.03 \\
\hline 17 & $2,3,4,5-\mathrm{TeCP}$ & Uncoupler & Non-covalent & $2.59( \pm 0.04)$ & $2.46( \pm 0.03)$ & $2.41( \pm 0.03)$ & 0.448 & -0.179 & 0.126 & 0 & -0.27 & 9.08 \\
\hline 18 & $2,3,4,6-\mathrm{TeCP}$ & Uncoupler $^{\mathrm{b}}$ & Non-covalent & $2.29( \pm 0.04)$ & $2.04( \pm 0.05)$ & $1.85( \pm 0.04)$ & 0.318 & -0.159 & 0.104 & 0 & -0.35 & 9.11 \\
\hline 19 & $2,3,5,6-\mathrm{TeCP}$ & Uncoupler & Non-covalent & $2.32( \pm 0.03)$ & $2.11( \pm 0.05)$ & $1.90( \pm 0.05)$ & 0.254 & -0.130 & 0.102 & 0 & -0.35 & 9.13 \\
\hline 20 & PCP & Uncoupler & Non-covalent & $3.03( \pm 0.04)$ & $2.96( \pm 0.01)$ & $2.91( \pm 0.02)$ & 0.515 & -0.171 & 0.135 & 0 & -0.28 & 9.09 \\
\hline 21 & 1,2,3-THB & Pro-electrophile & Covalent & $0.59( \pm 0.02)$ & $0.57( \pm 0.02)$ & $0.53( \pm 0.03)$ & 0.141 & -0.197 & 0.071 & 0 & -0.45 & 9.11 \\
\hline 22 & $\mathrm{HQ}$ & Pro-electrophile & Covalent & $2.56( \pm 0.05)$ & $2.52( \pm 0.03)$ & $2.51( \pm 0.02)$ & 0.097 & 0.059 & 0.049 & 0.042 & -0.41 & 9.04 \\
\hline 24 & TetraClHQ & Pro-redox cycler & Covalent & $1.68( \pm 0.03)$ & $1.66( \pm 0.04)$ & $1.64( \pm 0.01)$ & 0.104 & 0.091 & 0.066 & 0.036 & -0.43 & 9.09 \\
\hline 26 & 4-ClCat & Pro-electrophile & Covalent & $0.86( \pm 0.02)$ & $0.74( \pm 0.02)$ & $0.70( \pm 0.02)$ & 0.123 & -0.126 & 0.078 & 0 & -0.47 & 9.08 \\
\hline 27 & 3,5-DiClCat & Pro-electrophile & Covalent & $1.19( \pm 0.03)$ & $1.03( \pm 0.04)$ & $0.94( \pm 0.02)$ & 0.182 & -0.108 & 0.092 & 0 & -0.49 & 9.09 \\
\hline 28 & Res & Polar narcotic & Non-covalent & $-0.42( \pm 0.02)$ & $-0.42( \pm 0.02)$ & $-0.43( \pm 0.02)$ & 0.099 & -0.239 & 0.056 & 0 & -0.42 & 9.12 \\
\hline 30 & 4,6-DiClRes & Polar narcotic & Non-covalent & $0.51( \pm 0.02)$ & $0.49( \pm 0.02)$ & $0.49( \pm 0.02)$ & 0.146 & -0.254 & 0.064 & 0 & -0.43 & 9.13 \\
\hline \multicolumn{13}{|c|}{ Test set } \\
\hline 3 & $3-\mathrm{CP}$ & Polar narcotic & Non-covalent & $0.91( \pm 0.04)$ & $0.62( \pm 0.04)$ & $0.49( \pm 0.05)$ & 0.124 & -0.147 & 0.074 & 0 & -0.46 & 9.07 \\
\hline 9 & 3,4-DCP & Polar narcotic & Non-covalent & $1.43( \pm 0.04)$ & $1.35( \pm 0.03)$ & $1.35( \pm 0.02)$ & 0.253 & -0.178 & 0.088 & 0 & -0.40 & 9.04 \\
\hline 14 & $2,4,5-\mathrm{TCP}$ & Polar narcotic & Non-covalent & $1.99( \pm 0.03)$ & $1.90( \pm 0.04)$ & $1.73( \pm 0.05)$ & 0.199 & -0.166 & 0.090 & 0 & -0.35 & 9.09 \\
\hline 23 & ClHQ & Pro-electrophile & Covalent & $2.59( \pm 0.03)$ & $2.59( \pm 0.03)$ & $2.58( \pm 0.02)$ & 0.151 & 0.071 & 0.065 & 0.039 & -0.42 & 9.07 \\
\hline 25 & Cat & Pro-electrophile & Covalent & $0.73( \pm 0.03)$ & $0.67( \pm 0.02)$ & $0.60( \pm 0.02)$ & 0.107 & -0.104 & 0.057 & 0 & -0.47 & 9.09 \\
\hline 29 & 4-ClRes & Polar narcotic & Non-covalent & $0.16( \pm 0.03)$ & $0.04( \pm 0.03)$ & $0.00( \pm 0.03)$ & 0.133 & -0.264 & 0.061 & 0 & -0.45 & 9.15 \\
\hline
\end{tabular}

All MOA are the assigned mechanism of action according to Cronin et al. [14].

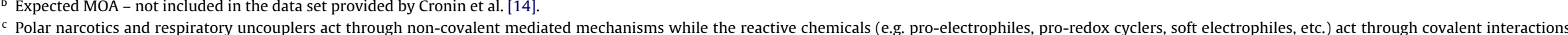
according to Schultz [31].

${ }^{d}$ Numbers in parenthesis refer to standard mean error of marine algal toxicity. 


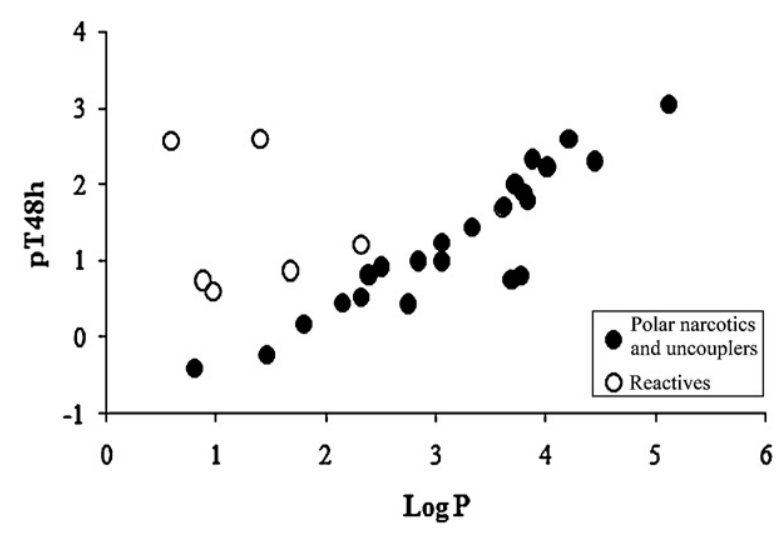

Fig. 1. Plot of marine algal toxicity $\left(p \mathrm{~T}_{48 \mathrm{~h}}\right)$ versus hydrophobicity described by Log P.

implicitly suggested that additional molecular descriptor(s) other than a hydrophobicity term would be required to accurately predict the toxicity of phenols towards $D$. tertiolecta. An initial modeling attempt was made using the pool of molecular descriptors including only 2D descriptors and Log P. However, all the resulting models had either low training set performance (i.e. $r^{2}<0.80$ ) or hydroquinones were found to be outliers. Following the removal of hydroquinones, the model presented in Eq. (6) resulted in a quality QSAR (which was developed using the training set in Table 1 without hydroquinones and its predictivity was assessed using compounds in the test set without hydroquinones):

$p \mathrm{~T}_{48}=0.63 \log \mathrm{P}+1.725$ MATS3p -0.38

$n_{t r}=22, \quad r^{2}=0.89, \quad r_{L O O}^{2}=0.80, \quad r_{L 2 O}^{2}=0.79, \quad R M S E_{t}=0.30 ;$

$\left.n_{\text {test }}=5, \quad Q_{\text {test }}^{2}=0.82, \quad R M S E_{p}=0.37\right)$

In Eq. (6), the Log P describes hydrophobicity while MATS3p is a 2D autocorrelation descriptor weighted by atomic polarizability (originally named as Moran autocorrelation lag three weighted by atomic polarizability) obtained from the Dragon 5.4 software. The positive contribution of this descriptor suggests that algal toxicity increases with increasing atomic polarizability of the phenol. Although this two-parameter QSAR produced highly satisfactory results, it was not capable of explaining the toxicity of hydroquinones. Therefore, to model the toxicity of phenols including hydroquinones to $D$. tertiolecta, 3D descriptors were included in the modeling process in search for global models which preferably did not produce any compound as outlier.

\subsection{Global models}

The European Centre for Ecotoxicology and Toxicology of Chemicals has published several reports $[8,28]$ to highlight the need for marine toxicity data and recently in the workshop on the probabilistic approaches to be used in marine risk assessment [11], experts have recognized the potential use of QSARs in efforts to fill the data gap present in this domain of ecotoxicology. One of the recommendations of the workshop was to develop models for compounds having similar mode of actions (i.e. local models). For regulatory usage, it is clearly important to develop and understand local models because this enables confidence to be better assigned to the predictions when these models are used in a real life scenario [29]. However, it should be kept in mind that the knowledge on the MOA of chemicals varies considerably [30] and assignment of the correct MOA is a difficult task [31]. Certain mechanisms may even be specific for the given species and these can be related to the metabolism and distribution of the compounds in vivo [3].
Therefore, as stated by Schultz [31], the models that accurately predict the toxicity of chemicals without first identifying their toxic mechanisms (i.e. global models), are highly desirable.

In the present study, three global models were found to display acceptable training $\left(r^{2} \geq 0.80, r_{L O O}^{2}\right.$ and $\left.r_{L 2 O}^{2} \geq 0.70\right)$ and test set $\left(Q_{\text {test }}^{2} \geq 0.70\right)$ statistics and these are listed in Table 2 together with the statistics of the consensus MLR model.

The models in Table 2 were also subjected to the predictivity criteria proposed by Golbraikh and Tropsha [24] as summarized in Eqs. (2)-(4) and also to $Y$-randomization (Table 3).

It was found that $r_{r}^{2}$ of the randomized models were lower than the corresponding $r^{2}$ of the non-randomized (i.e. original) model and the $R_{p}^{2}$ values were higher than 0.5 . This finding indicated that the obtained relationships were not due to chance. At the end of the rigorous validation process, we were convinced that the proposed models were robust, predictive and not chance correlations.

It should be noted that the consensus model integrating all validated individual models was found to be the most externally predictive. This finding indicates that consensus modeling approach can be used to predict the toxicity of phenols towards D. tertiolecta. Previously, Zhu et al. [27] reached a similar conclusion in their efforts to offer global QSARs to predict the toxicity of a large set of phenols towards Tetrahymena pyriformis. They noted that the consensus models offer better predictivity than the individual models, which was similar to our finding for the relatively small marine algal toxicity data set.

\subsection{The role of descriptors}

The descriptors that appeared in the linear QSARs (Table 2) involve Mor24m (3D-MoRSE - signal 24/weighted by atomic masses), Mor24p (3D-MoRSE - signal 24/weighted by atomic polarizabilities) and Mor18u (3D-MoRSE - signal 18/unweighted) obtained from the Dragon software. Another Dragon descriptor, namely the HATS7e (Leverage weighted autocorrelation of lag 7/weighted by atomic Sanderson electronegativities) is a GEometry, Topology, and Atom-Weights AssemblY (GETAWAY) descriptor which encode both the geometrical information provided by the influence molecular matrix and the topological information provided by the molecular graph, weighted by chemical information encoded in selected atomic weightings [32]. The descriptors that were obtained from the CODESSA software were $C_{\text {ortho }}$ (originally named as MOPAC partial charges for atom \# $0000002-C)$ and $k_{1} \mathrm{max}^{\mathrm{a}}$, which indicate the partial charge on the second atom (carbon) located at the (2-) position relative to the functional group (i.e., the $\mathrm{OH}$ moiety) and the maximum atomic force constant, respectively. For the latter, Katritzky et al. [33] stated that this descriptor can be associated with intermolecular interactions.

The commonality of the three QSARs developed in this study is the presence of 3D-MoRSE descriptors (Table 2). These descriptors provide information of a compound from its three-dimensional structure using a molecular transform derived from an equation used in electron diffraction studies and by taking several atomic properties into account they have the potential to encode highly flexible representations of a molecule [34]. The presence of Mor24m (3D-MoRSE signal 24/weighted by atomic masses) and Mor24p (3D-MoRSE signal 24/weighted by atomic polarizabilities) in two distinct QSARs (model 1 and model 2) suggests that biological activity has a significant dependence on the size and polarizability of the molecules. On the other hand, the presence of Mor18u (3D-MoRSE - signal 18/unweighted), which is neither influenced by the atomic polarizability nor by the atomic mass of phenols, suggests that the 3D structure of the molecules might have an impact on their toxicity as well. 
Table 2

QSARs that displayed acceptable training and test set statistics.

\begin{tabular}{|c|c|c|c|c|c|c|c|c|c|}
\hline \multirow[t]{2}{*}{ Model ID } & \multirow[t]{2}{*}{ MLR models } & \multicolumn{5}{|c|}{ Training set } & \multicolumn{3}{|c|}{ Test set } \\
\hline & & $n$ & $r^{2}$ & $r_{L O O}^{2}$ & $r_{L 2 O}^{2}$ & $R M S E_{t}$ & $n$ & $Q_{\text {test }}^{2}$ & $R M S E_{p}$ \\
\hline 1 & $p \mathrm{~T}_{48 \mathrm{~h}}=6.18$ Mor $24 \mathrm{~m}+6.27 C_{\text {ortho }}+1.06$ & 24 & 0.82 & 0.74 & 0.74 & 0.38 & 6 & 0.85 & 0.34 \\
\hline 2 & $p \mathrm{~T}_{48 \mathrm{~h}}=31.10$ Mor $24 \mathrm{p}+44.41$ HATS7e -1.36 & 24 & 0.83 & 0.71 & 0.70 & 0.36 & 6 & 0.88 & 0.31 \\
\hline \multirow[t]{2}{*}{3} & $p \mathrm{~T}_{48 \mathrm{~h}}=11.21$ Mor $18 \mathrm{u}+4.37 C_{\text {ortho }}-8.66 \mathrm{k}_{1} \mathrm{max}^{\mathrm{a}}+85.13$ & 24 & 0.80 & 0.73 & 0.70 & 0.39 & 6 & 0.92 & 0.26 \\
\hline & MLR Consensus Model ${ }^{\mathrm{a}}$ & 24 & 0.88 & 0.86 & 0.85 & 0.31 & 6 & 0.94 & 0.23 \\
\hline
\end{tabular}

a Average of the three models (Models 1, 2, and 3).

\subsection{Applicability domain of the MLR models}

The applicability domain of each model was constructed using the plot of standardized residuals against leverages (Fig. 2).

All compounds had standardized residuals $<2.5$ indicating that there was not a response outlier (Y outliers) in the developed QSARs. The cut-off hat value for model 1, model 2, and model 3 were found to be $0.375,0.375$, and 0.5 , respectively. On the basis of these cut-off values, tetrachlorohydroquinone ( $\mathrm{ID}=24)$ was detected as an X outlier for model 1 (Fig. 2a). For model 2, both tetrachlorohydroquinone and hydroquinone ( $I D=22$ ) were $\mathbf{X}$ outliers (Fig. 2b); while there was not any $\mathbf{X}$ outlier for model 3 (Fig. 2c).

\subsection{Counter Propagation Artificial Neural Networks (CP ANNs)}

It can be assumed that the consensus MLR model (Table 2) carries significant toxicological information that is encoded in the descriptors that appeared in the three MLR models. In an effort to understand how these significant descriptors would contribute to a non-linear model, we initially attempted to construct a $\mathrm{CP}$ ANN using all six molecular descriptors that appeared in the proposed linear models (Mor24m, $C_{\text {ortho }}$, Mor24p, HATS7e, Mor18u, and $k_{1} \max ^{\mathrm{a}}$ ). However, it was found that Mor24m and Mor24p were inter-correlated; therefore, the former was removed from analysis while the latter was kept in as it had higher correlation to the toxicity. Likewise, $C_{\text {ortho }}$ and HATS7e were also found to be inter-correlated and the latter was removed from modeling due to the presence of zero values for most of the compounds.

With the introduction of four descriptors as input variables, the four-parameter CP ANN modeling was performed employing three different network architectures $(5 \times 5,6 \times 6$ and $7 \times 7)$ to cover all investigated objects as well as a range of learning iterations (10-400 iterations). The modeling was performed on the training set (24 compounds), employing the leave-one-out cross-validation procedure for optimization of the technical parameters. The best CP ANN model was selected according to the highest $r_{L O O}^{2}$ value which was obtained from a network of $6 \times 6$ dimensions and 350 epochs.

The results revealed that the statistical parameters of the fourparameter CP ANN model were found to be comparable to the consensus MLR model (Table 4 and Fig. 3).

These results apparently show the good predictive ability of the CP ANN model for prediction of the marine algal toxicity of phenols to D. tertiolecta. Clearly, the four-parameter CP ANN model should not be read strictly as a non-linear counterpart of the consensus MLR model. Yet, it provides useful information regarding the descriptors or descriptor combinations used in the present study (Fig. 4). Additionally, the CP ANN model enabled a successful clustering of the phenols with similar biological toxicity as well as interaction type over the entire network (Fig. 4E).

The visual analysis of the output layers for the obtained CP ANN model (Fig. 4) highlighted the good agreement between the weight maps for the 3D-MoRSE descriptors and toxicity. Mor24p (Fig. 4C) and Mor18u (Fig. 4D) displayed very similar color distribution to that of the biological toxicity (Fig. 4E) which accounts for a good positive correlation for almost all investigated compounds, and especially for the chlorophenols. On the other hand, the weights for $C_{\text {ortho }}$ (Fig. 4A) and $k_{1}$ max $^{\mathrm{a}}$ (Fig. 4B), respectively, follow a different color distribution compared to the biological toxicity map (Fig. 4E). $C_{\text {ortho }}$ molecular descriptor can be regarded as an accountant parameter for good separation of two classes: hydroquinones $(\mathrm{ID}=22,23,24)$ and resorcinols (ID $=28,29,30)$; while $k_{1} \max ^{\mathrm{a}}$ molecular descriptor clearly separates the catechols (ID $=25,26$, 27 ). Note that the descriptor's values in Fig. 4 (A, B, C, and D) are normalized between 0.00 (minimum value) and 1.00 (maximum value). The output layer for 48 -h toxic exposition $\left(p \mathrm{~T}_{48 \mathrm{~h}}\right)$ is presented as E, where the values 0.05 and 2.81 correspond to minimum and maximum toxicity predicted by the $\mathrm{CP}$ ANN model, respectively. The numbers in the plot (E) correspond to the positions of the compounds from the training (white) and test set (black) in the top-map of the Kohonen layer.

\subsection{Subset models and mechanistic interpretation}

In an effort to better understand the role of 3D-MoRSE descriptors and interpret the developed MLR models mechanistically, the whole dataset was decomposed into several subsets according to (a) the structure of the compounds (i.e. simple phenols and polyphenols) and (b) the nature of mechanisms (i.e. non-covalent and covalent) as described by Schultz [31]. As the applicability domains of model 1 and model 2 suggested, the presence of hydroquinones influenced these global QSARs; therefore, the subset analyses using 3D-MoRSE descriptors were also carried out for subgroups excluding hydroquinones (Table 5).

The analyses revealed that the 3D-MoRSE descriptors were able to predict the toxicity of chlorophenols (Table 5, Subset ID 1 ) whereas they failed to do so for polyphenols (Table 5, Subset ID 2) even when hydroquinones were removed (Table 5, Subset ID 3). This is not unexpected because polyphenols are involved in different reactivity mechanisms and a structure basedclassification would be futile in efforts to predict the toxicity of

Table 3

Additional external validation criteria and $Y$-randomization.

\begin{tabular}{|c|c|c|c|c|c|c|c|}
\hline \multirow[t]{2}{*}{ Model ID } & \multicolumn{5}{|c|}{ Criteria by Golbraikh and Tropsha [24] } & \multicolumn{2}{|c|}{$Y$-randomization } \\
\hline & $\frac{R^{2}-R_{0}^{2}}{R^{2}}<0.1$ & $0.85 \leq K \leq 1.15$ & $\frac{R^{2}-R_{0}^{\prime 2}}{R^{2}}<0.1$ & $0.85 \leq K^{\prime} \leq 1.15$ & $\left|R_{0}^{2}-R_{0}^{\prime 2}\right| \leq 0.3$ & $r_{r}^{2}$ & $R_{P}^{2}>0.5$ \\
\hline 1 & 0.046 & 0.893 & 0.001 & 1.068 & 0.038 & 0.09 & 0.70 \\
\hline 2 & 0.023 & 0.872 & 0.001 & 1.109 & 0.020 & 0.09 & 0.71 \\
\hline 3 & 0.007 & 0.913 & 0.025 & 1.067 & 0.016 & 0.12 & 0.66 \\
\hline MLR Consensus & 0.005 & 0.892 & 0.001 & 1.105 & 0.004 & - & - \\
\hline
\end{tabular}



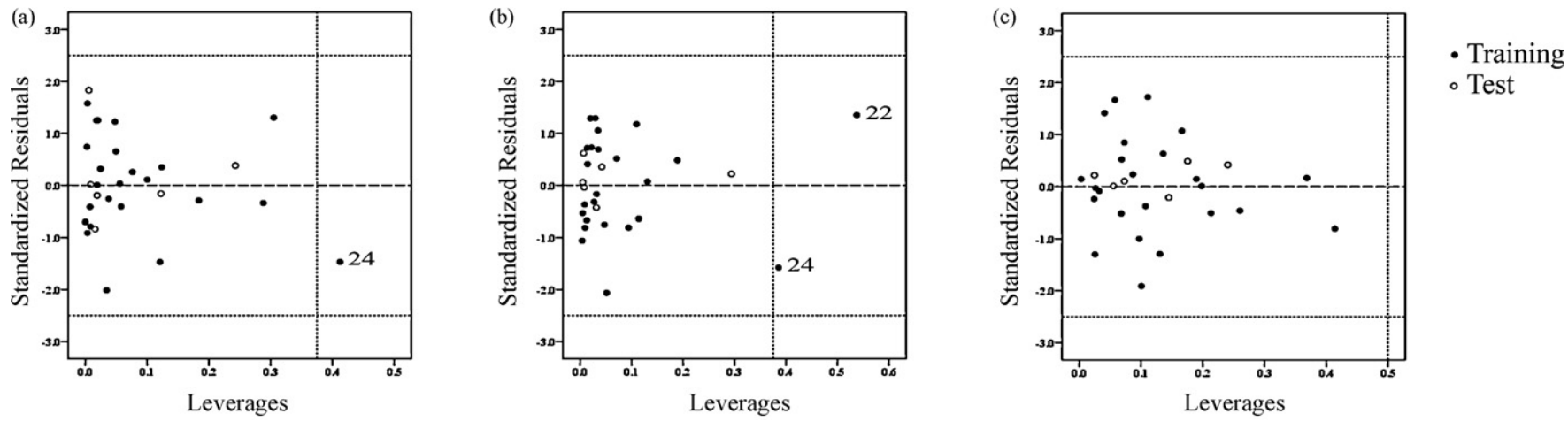

Fig. 2. Applicability domains of (a) model 1, (b) model 2 and (c) model 3.

Table 4

Statistical comparison of the MLR consensus and CP ANN models.

\begin{tabular}{|c|c|c|c|c|c|c|c|}
\hline \multirow[t]{2}{*}{ Model } & \multicolumn{4}{|c|}{ Training set } & \multicolumn{3}{|c|}{ Test set } \\
\hline & $n$ & $r^{2}$ & $r_{\mathrm{LOO}}^{2}$ & $R M S E_{t}$ & $n$ & $Q_{\text {test }}^{2}$ & $R M S E_{p}$ \\
\hline MLR Consensus & 24 & 0.88 & 0.86 & 0.31 & 6 & 0.94 & 0.23 \\
\hline CP ANN & 24 & 0.92 & 0.73 & 0.26 & 6 & 0.93 & 0.25 \\
\hline
\end{tabular}
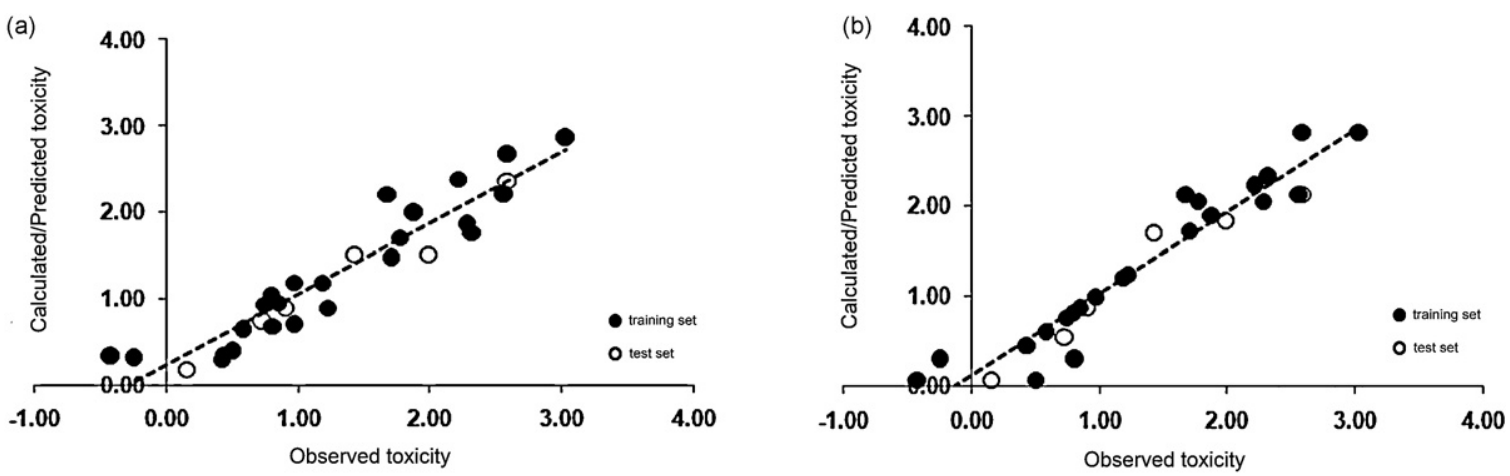

Fig. 3. Relationships between the calculated/predicted and observed ( $p T_{48 h}$ ) toxicity for (a) MLR consensus and (b) CP ANN models.

poly-hydroxylated aromatics. However, since chlorophenols have structural as well as mechanistic similarity, all the 3D-MoRSE descriptors, particularly Mor24p, was found to be in very good agreement with this class of compounds (Table 5, Subset ID 1: $r^{2}=0.88, r_{L O O}^{2}=0.85$ ), as well as with compounds eliciting toxic effects through non-covalent mediated interactions (Table 5, Subset ID 4: $r^{2}=0.85, r_{L O O}^{2}=0.83$ ). Closer inspection of the Mor24p (Table 1 ) revealed that chlorophenols containing chlorine atoms at 2 - and 6-positions have significantly lower values compared to the congeners with the same number of chlorine atoms. Note that the ortho substituted chlorophenols were found to be less toxic than the meta and para substituted congeners also (Table 1). As stated by Boyd et al. [35], the lower toxicity of ortho substituted chlorophenols has been ascribed to the shielding of the $\mathrm{OH}$ group by chlorine atom(s). Mor24m was also found to be useful in predicting the toxicity of chlorophenols (Table 5, Subset ID 1: $r^{2}=0.80, r_{L O O}^{2}=0.76$ ); however, considering the superiority of Mor24p over Mor24m, it might be inferred that 3D information weighted by atomic polarizability might be more important in the toxicity of chlorophenols to $D$. tertiolecta as compared to that weighted by atomic mass.

It was interesting to note that Mor24p was alone able to explain $84 \%$ of the variance in the data set when hydroquinones were

Table 5

Correlation of 3D-MoRSE descriptors with the toxicity of phenols to Dunaliella tertiolecta.

\begin{tabular}{|c|c|c|c|c|c|c|c|c|}
\hline \multirow[t]{2}{*}{ Subset ID } & \multirow[t]{2}{*}{ Subset specification } & \multirow[t]{2}{*}{$n$} & \multicolumn{2}{|c|}{ Mor24m } & \multicolumn{2}{|c|}{ Mor24p } & \multicolumn{2}{|c|}{ Mor18u } \\
\hline & & & $r^{2}$ & $r_{L O O}^{2}$ & $r^{2}$ & $r_{L O O}^{2}$ & $r^{2}$ & $r_{L O O}^{2}$ \\
\hline 1 & Phenol + chlorophenols & 20 & $0.80^{\mathrm{a}}$ & 0.76 & 0.88 & 0.85 & 0.78 & 0.73 \\
\hline 2 & Polyphenols & 10 & 0.00 & n.t. & 0.00 & n.t. & 0.06 & n.t. \\
\hline 3 & Polyphenols (except hydroquinones) & 7 & 0.43 & 0.03 & 0.59 & 0.30 & 0.74 & 0.46 \\
\hline 4 & Non-covalent & 23 & 0.72 & 0.68 & 0.85 & 0.83 & 0.73 & 0.68 \\
\hline 5 & Covalent & 7 & 0.02 & n.t. & 0.20 & n.t. & 0.56 & 0.22 \\
\hline \multirow[t]{3}{*}{6} & Covalent (except hydroquinones) & 4 & 0.51 & 0.00 & 0.64 & 0.05 & 0.97 & 0.92 \\
\hline & All phenols & 30 & 0.50 & 0.46 & 0.49 & 0.42 & 0.49 & 0.43 \\
\hline & All phenols (except hydroquinones) & 27 & 0.72 & 0.69 & 0.84 & 0.82 & 0.65 & 0.59 \\
\hline
\end{tabular}

n.t., not tested due to low $r^{2}$.

a Relationships with $r^{2}$ and $r_{c v}^{2} \geq 0.70$ are highlighted in bold. 
A

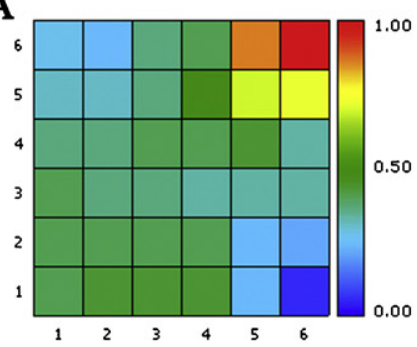

C

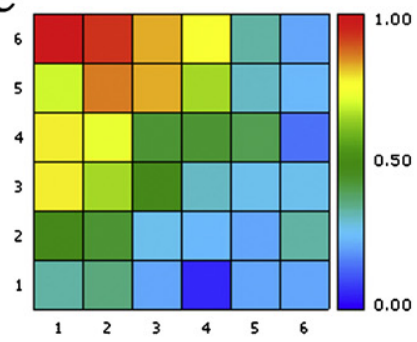

B

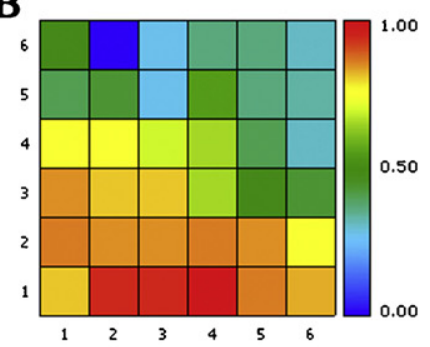

D

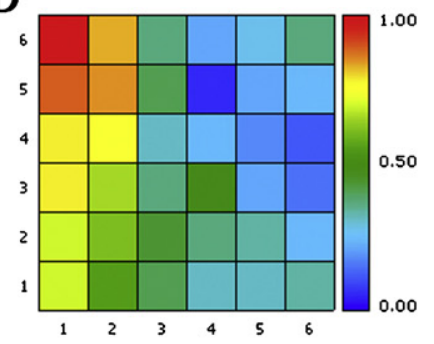

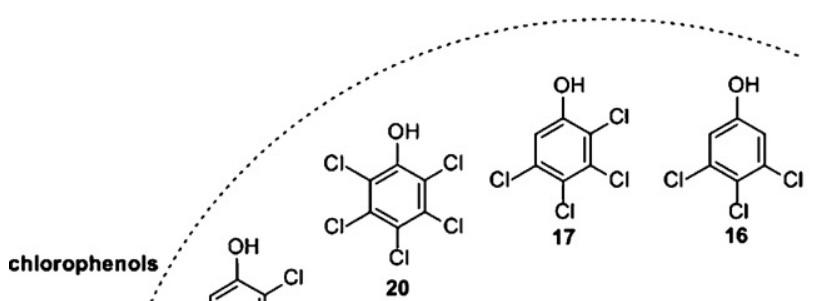

E

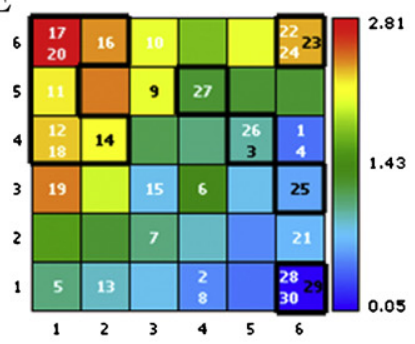

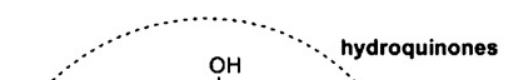<smiles>Oc1ccc(O)cc1</smiles><smiles>Oc1c(Cl)c(O)c(Cl)c(Cl)c1Cl</smiles>

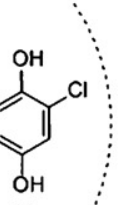<smiles>Cc1cc(Cl)c(O)cc1Cl</smiles><smiles>Oc1ccc(O)c(Cl)c1</smiles><smiles>Oc1ccccc1O</smiles>

Fig. 4. Output layers of the selected four-parameter CP ANN model. (A) $C_{\text {ortho }}$; (B) $k_{1}$ max $^{\mathrm{a}}$; (C) Mor24p; (D) Mor18u; (E) $p \mathrm{~T}_{48 \mathrm{~h}}$ and descriptor-based clustering of phenols over the CP ANN network (compound ID in white and black refer to training and test set compounds, respectively).

removed from analysis (Table 5). The high correlation of Mor24p with phenols except hydroquinones implicitly suggests that the presence of the additional descriptor (HATS7e) in the developed QSAR (model 2, Table 2) is mainly to account for the toxicity of hydroquinones. In line with this expectation, closer inspection of HATS7e revealed that this descriptor has values other than zero only for hydroquinones (Table 1 ). The concomitance of Mor24m and $C_{\text {ortho }}$ can also be interpreted similarly for model 1 (Table 2). Inspection of $C_{\text {ortho }}$ revealed distinct values for the three hydroquinones in that only these compounds had positive partial charges in the second carbon atom (Table 1). Constitution of model 3 (Table 2), on the other hand, differs from the other models in that there are two additional descriptors (i.e. $C_{\text {ortho }}$ and $k_{1} \mathrm{max}^{\mathrm{a}}$ ). Note that Mor $18 \mathrm{u}$ was shown to be correlated very strongly with the toxicity of phenols acting through covalent interactions except hydroquinones (Table 5, Subset ID 6: $r^{2}=0.97, r_{L O O}^{2}=$ 0.92 ). Considering the relatively good agreement of this descriptor with compounds acting through non-covalent interactions also (Table 5, Subset ID 4: $r^{2}=0.72, r_{L O O}^{2}=0.68$ ), the presence of $C_{\text {ortho }}$ and $k_{1} \mathrm{max}^{\mathrm{a}}$ can be said to explain the remaining variance in the toxicity, particularly that inflicted by compounds acting through covalent mediated mechanisms. 
Table 6

External prediction performance of the proposed QSARs.

\begin{tabular}{|c|c|c|c|c|c|c|c|c|c|c|c|c|}
\hline \multicolumn{8}{|c|}{ External set statistics } & \multicolumn{5}{|c|}{ Criteria by Golbraikh and Tropsha [24] } \\
\hline Model & $\begin{array}{l}\text { Number of } \\
\text { external set } \\
\text { compounds }\end{array}$ & $\begin{array}{l}\text { Compounds } \\
\text { out of AD } \\
\left(h>h^{*}\right)\end{array}$ & $\begin{array}{l}\text { Number of } \\
\text { response } \\
\text { outliers }\end{array}$ & $n^{\mathrm{a}}$ & $Q_{e x t}^{2}$ & $R M S E_{\text {ext }}$ & $\%$ Coverage ${ }^{b}$ & $\frac{R^{2}-R_{0}^{2}}{R^{2}}<0.1$ & $0.85 \leq K \leq 1.15$ & $\frac{R^{2}-R_{0}^{\prime 2}}{R^{2}}<0.1$ & $0.85 \leq K^{\prime} \leq 1.15$ & $\left|R_{0}^{2}-R_{0}^{\prime 2}\right| \leq 0.3$ \\
\hline Model 1 & 44 & 1 & 4 & 40 & 0.74 & 0.47 & 97 & 0.06 & 1.04 & 0.05 & 0.90 & 0.01 \\
\hline Model 2 & 44 & 14 & 7 & 23 & 0.69 & 0.49 & 68 & 0.07 & $1.20^{c}$ & 0.01 & 0.78 & 0.05 \\
\hline Model 3 & 44 & 26 & 5 & 13 & 0.79 & 0.41 & 41 & 0.04 & 1.05 & 0.12 & 0.88 & 0.07 \\
\hline
\end{tabular}

a Number of compounds put into analysis after the removal of response outliers and compounds out of AD which were poorly predicted by the model.

b Ratio of the number of external set compounds within the model AD to the total number of compounds in the external set, expressed as percentage.

${ }^{c}$ Criteria that were not met by the model were highlighted in bold.

From an organic chemistry perspective, hydroquinones can undergo one-electron oxidation to semi-quinone, and being a radical anion the semi-quinone can be further oxidized to benzoquinone. Alternatively, hydroquinone can be oxidized directly to benzoquinone in a two-electron transfer reaction [3]. As stated by Aptula et al. [36] hydroquinone is usually considered to be a pro-electrophilic sensitizer that can be oxidized either abiotically or enzymatically to benzoquinone which is a chemically reactive Michael type acceptor electrophile. The researchers also noted that benzoquinone itself is recognized as an extreme sensitizer, and guinea pig cross-challenges pointed out that hydroquinone sensitizes via benzoquinone. Satoh et al. [37] also indicated that hydroquinones are not themselves electrophilic and require oxidative conversion to their quinone forms to be involved in electrophilic processes. On the basis of the explanations above, the reason behind the excess toxicity of hydroquinones (particularly hydroquinone and chlorohydroquinone) towards $D$. tertiolecta possibly lies in the transformation of these compounds into their more electrophilic quinone forms. Previously, Devillers et al. [38] found that $72 \mathrm{~h} \mathrm{IC} 50$ value of hydroquinone towards freshwater algae $S$. capricornutum was $0.34 \mathrm{mg} / \mathrm{L}$, which is very close to $72 \mathrm{~h} \mathrm{IC} 50$ value determined in this study $(0.33 \mathrm{mg} / \mathrm{L})$. Almost similar $\mathrm{IC}_{50}$ values determined in tests using two different algal species suggest that toxicity mechanism of hydroquinone towards algae might be similar.

The information regarding the excess toxicity of hydroquinones was best captured by HATS7e and $C_{\text {ortho }}$ in the present study. However, the fact that both descriptors were able to discriminate excess hydroquinone toxicity should not be interpreted as the ability of these descriptors in describing the reactivity of phenols. The information captured by both descriptors, particularly by $C_{\text {ortho }}$, can be interpreted as the propensity of a pro-electrophile to undergo oxidation reactions and transform into its more electrophilic form or, alternatively, the propensity of a compound to interact with nucleophilic target sites at this particular carbon atom. Additionally, the low $C_{\text {ortho }}$ values of resorcinols, which is also reflected in the weight map of this descriptor (Fig. 4A), indicate that this class of compounds may not be active in electrophilic/nucleophilic processes in the marine algal test system. This finding is in line with the organic chemistry principle that resorcinols are not involved in oxidation reactions unless activated by additional groups $[3,39]$.

The difficulties in modeling the toxicity of compounds that may act either directly or indirectly as electrophiles have been reported in various studies $[14,29]$. The difficulty in modeling the toxicity of such compounds might be due to the fact that aromatic compounds are hydroxylated by intracellular monooxygenases in vivo, and it is, therefore, difficult to discriminate the effect of initial compound and its metabolites [39]. Additionally, from a statistical perspective, the dominance in the data set of compounds acting through similar MOAs (i.e. compounds acting through non-covalent MOAs like polar narcosis and respiratory uncoupling) results in the selection of a descriptor, or a set of descriptors, that best explain these mechanisms [29]. However, compounds acting through other MOAs and consequently the associated descriptors may not be equally represented in the models purely due to issues related to the data set bias. For instance, the marine algal data set presented in this study includes chlorophenols and resorcinols, which act through non-covalent mediated interactions; therefore, the marine algal QSARs can be expected to explain the toxicity of these compounds first to be able to explain the dominant part of the variance. Indeed, the developed QSARs included 3D-MoRSE descriptors which were able to explain a great part of the variance in the toxicity of non-covalent mediated toxicity (Table 5, Subset ID 4). The remaining descriptors were likely to be selected in the models mainly to account for the variance in the toxicity of compounds acting through covalent interactions, particularly hydroquinones. Nevertheless, when coupled with 3D-MoRSE descriptors, they resulted in the development of QSARs with acceptable training and test set statistics, indicating that they, particularly $C_{\text {ortho }}$, at least encode adequate information to predict the toxicity of phenols presented in this study towards marine alga $D$. tertiolecta.

\subsection{External predictivity of the proposed QSARS}

It is of our interest to further test the predictivity of the proposed QSARs by evaluating their performance in predicting the toxicity of a heterogeneous data set obtained from literature [17]. The external prediction performance of the proposed models can be seen in Table 6 . The descriptor values for the external set compounds are provided as supplementary material to this study (Table S2).

As indicated by Kar and Roy [23], $Q^{2}$ values higher than 0.5 are considered acceptable in terms of a model's external predictivity. Accordingly, following the removal of four outliers, the predictive performance of model 1 (Table 2 ) was found to be very good $\left(Q_{\text {ext }}^{2}=0.74\right.$, Table 6$)$, especially considering its excellent coverage (97\%, Table 6) of the external data set. On the other hand, model 2 and in particular model 3 had narrow applicability domains which were reflected in low coverage of the external dataset by these models (68\% and 41\%, respectively, Table 6). Further evaluation of the proposed QSARs according to the external prediction criteria put forward by Golbraikh and Tropsha [24] revealed that while model 1 met all the criteria, model 2 and model 3 failed to concur with some of the requirements (Table 6). The 4-parameter CPANN, which was built using all the descriptors appearing in model 3 plus another one from model 2 (i.e. Mor24p), had also low predictivity and coverage (results not shown).

Of the proposed models, model 1 was highlighted because of its overall performance. The Williams plot and the observed versus calculated/predicted toxicity for model 1 are illustrated in Fig. 5 a and $b$, respectively. The predicted toxicities and leverages by model 1 for the external set compounds are provided as supplementary material to this study (Table S3).

Since the external data set obtained from Furusjö et al. [17] is a diverse data set which is comprised of experimental toxicity 

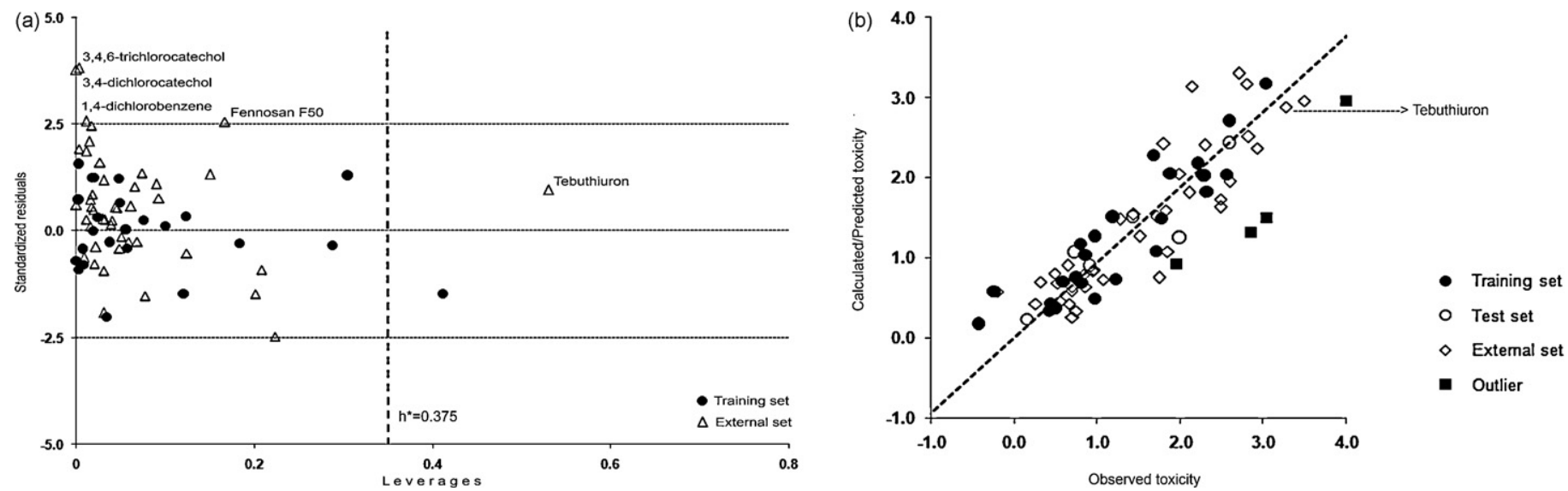

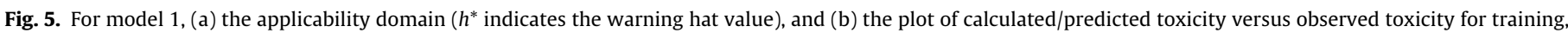
test and external sets.

data generated in different laboratories, it is hardly possible to predict the toxicity of every compound accurately. As can be seen from Fig. 5 a, four outliers, namely, 3,4-dichlorocatechol, 3,4,6trichlorocatechol, 1,4-dichlorobenzene and Fennosan F50 were found to be more toxic than predicted by model 1 . Following the exclusion of these four outliers from analysis, the external predictivity of model 1 was found to be highly satisfactory. It is important to note that only Tebuthiuron was found to be a structurally high leverage compound in the Furusjö data set (Fig. 5a). However, its toxicity was well-predicted by model 1 as indicated by the small residual of this compound (Fig. 5b). Note that this compound is the only chemical in the data set which contains sulfur in its structure.

\section{Conclusion}

This study presented for the first time in its entirety the toxicity of 30 phenols towards $D$. tertiolecta. It was found that the marine algal toxicity of polar narcotics and respiratory uncouplers was strongly correlated to hydrophobicity as described by Log P. Compounds expected to act by more reactive mechanisms, particularly hydroquinones, displayed toxicity in excess of that predicted by $\log P$.

In an attempt to model the toxicity of phenols to $D$. tertiolecta using only 2D descriptors and Log P, a quality QSAR employing the hydrophobicity parameter $(\log P)$ and MATS3p, the latter being a $2 \mathrm{D}$ autocorrelation descriptor weighted by atomic polarizability, was obtained only after the removal of hydroquinones from the data set. Following the inclusion of $3 \mathrm{D}$ descriptors in the modeling process, three global QSARs were developed using MLR. It was demonstrated that global model statistics were improved by employing the consensus modeling approach where the predictions from individual models were averaged. Another significant result was obtained from non-linear modeling of the data set. The four-parameter CP ANN model, which was constructed using four out of six descriptors that appeared in the linear models, matched the external predictivity of the consensus MLR model.

The analyses made on subsets based upon the nature of mechanisms involved in the whole data set (i.e. non-covalent and covalent mediated mechanisms) revealed that 3D-MoRSE descriptors, particularly the one weighted by atomic polarizability (i.e. Mor24p), was highly correlated to the marine algal toxicity of phenols except hydroquinones. This finding suggested that the toxicity of phenols to marine alga $D$. tertiolecta was mainly controlled by the atomic polarizability and 3D structure of the molecule. Hydroquinones, which are believed to act through reactive mechanisms of toxic action towards $D$. tertiolecta test system, were highlighted as influential compounds in the developed QSARs.

Further validation of the proposed algal QSARs was attempted using an external set containing the toxicity data of a diverse set of chemicals towards freshwater alga $P$. subcapitata. Although the global QSARs were developed using toxicity data on marine alga $D$. tertiolecta, the external prediction performance of one of the global models (model 1) was particularly promising considering the differences between the biological constitution of each organism as well as the differences in test setups (e.g. light intensity, temperature, etc.) and algal growth medium characteristics (e.g. $p \mathrm{H}$, salinity, chemical composition, etc.).

\section{Acknowledgements}

The financial support of Bogazici University Bilimsel Araştırma Projeleri (Project No: 5564) is gratefully appreciated. Also, the authors would like to thank to the Scientific and Technological Research Council of Turkey (TUBITAK-Project No: 108Y119) and Slovenian Ministry for Higher Education, Science and Technology (Grant P1-0017).

\section{Appendix A. Supplementary data}

Supplementary data associated with this article can be found, in the online version, at http://dx.doi.org/10.1016/j.jmgm. 2012.06.002.

\section{References}

[1] P. Duchowicz, A.G. Mercader, F.M. Fernandez, E.A. Castro, Prediction of aqueous toxicity for heterogeneous phenol derivatives by QSAR, Chemometrics and Intelligent Laboratory Systems 90 (2008) 97-107.

[2] United States Environmental Protection Agency, Ambient water quality criteria for chlorinated phenols, Washington, DC, USA, 1980.

[3] A.O. Aptula, D.W. Roberts, M.T.D. Cronin, T.W. Schultz, Chemistry-toxicity relationships for the effects of di- and trihydroxybenzenes to Tetrahymena pyriformis, Chemical Research in Toxicology 18 (2005) 844-854.

[4] J. Jensen, Chlorophenols in the terrestrial environment, Reviews of Environment Contamination and Toxicology 146 (1996) 25-51.

[5] TerraTox ${ }^{\mathrm{TM}}$ database, Version 3.0, Terrabase, Inc., Hamilton, Ontario, Canada, 2006.

[6] M.E. DeLorenzo, Utility of dunaliella in ecotoxicity testing, in: A. Ben-Amotz, J.E.W. Polle, D.V.S. Rao (Eds.), The Alga Dunaliella: Biodiversity, Physiology, Genomics and Biotechnology, Science Publishers, Enfield, NH, 2009, pp. 495-512.

[7] M.T.D. Cronin, T.I. Netzeva, J.C. Dearden, R. Edwards, A.D.P. Worgan, Assessment and modeling of the toxicity of organic chemicals to Chlorella vulgaris: development of a novel database, Chemical Research in Toxicology 17 (2004) 545-554. 
[8] European Centre for Ecotoxicology and Toxicology of Chemicals, Risk Assessment in Marine Environments, Technical Report No. 82, Brussels, Belgium, 2001.

[9] T.H. Hutchinson, N. Scholz, W. Guhl, Analysis of the ECETOC Aquatic Toxicity (EAT) database IV - comparative toxicity of chemical substances to freshwater versus saltwater organisms, Chemosphere 36 (1998) 143-153.

[10] J.R. Wheeler, K.M. Leung D. Morritt, N. Sorokin, H. Rogers, R. Toy, H. Martin, P. Whitehouse, M. Crane, Freshwater to saltwater toxicity extrapolation using species sensitivity distributions, Environmental Toxicology and Chemistry 21 (2002) 2459-2467

[11] European Centre for Ecotoxicology and Toxicology of Chemicals, Workshop on the probabilistic approaches for marine hazard assessment, Workshop Report No. 15, Brussels, Belgium, 2008.

[12] American Public Health Association, American Water Works Association, and Water Environment Federation, Standard Methods for the Examination of Water and Wastewater, 20th ed., Washington, DC, USA, 1998.

[13] Organization for Economic Co-operation and Development, Guideline 201: Freshwater Alga and Cyanobacteria Growth Inhibition Test, Paris, France, 2006.

[14] M.T.D. Cronin, A.O. Aptula, J.C. Duffy, T.I. Netzeva, P.H. Rowe, I.V. Valkova, T.W. Schultz, Comparative assessment of methods to develop QSARs for the prediction of the toxicity of phenols to Tetrahymena pyriformis, Chemosphere 49 (2002) 1201-1221.

[15] S.P. Bradbury, R.L. Lipnick, Introduction: structural properties for determining mechanisms of toxic action, Environmental Health Perspectives 87 (1990) $181-182$.

[16] Organization for Economic Co-operation and Development, Guidance document on the validation of (quantitative) structure-activity relationships [(Q)SAR] Models, OECD Environment Health and Safety Publications, Series on Testing and Assessment No. 69. Paris, France, 2007.

[17] E. Furusjö, A. Svenson, M. Rahmberg, M. Andersson, The importance of outlier detection and training set selection for reliable environmental QSAR predictions, Chemosphere 63 (2006) 99-108.

[18] M.D. Ertürk, M.T. Saçan, First toxicity data of chlorophenols on marine alga Dunaliella tertiolecta: correlation of marine algal toxicity with hydrophobicity and interspecies toxicity relationships, Environmental Toxicology and Chemistry 31 (2012) 1113-1120.

[19] N. Minovski, DragCOD v1.0, laboratory for chemometrics, National Institute of Chemistry, Ljubljana, 2009.

[20] A.R. Katritzky, V.S. Lobanov, M. Karelson, CODESSA, Training Manual, Gainsville, 1995.

[21] J. Zupan, J. Gasteiger, Neural Networks in Chemistry and Drug Design, WileyVCH, Weinheim, 1999.

[22] V. Consonni, D. Ballabio, R. Todeschini, Comments on the definition of the $Q^{2}$ parameter for QSAR validation, Journal of Chemical Information and Modeling 49 (2009) 1669-1678.

[23] S. Kar, K. Roy, First report on interspecies quantitative correlation of ecotoxicity of pharmaceuticals, Chemosphere 81 (2010) 738-747.

[24] A. Golbraikh, A. Tropsha, Beware of q2!, Journal of Molecular Graphics and Modelling 20 (2002) 269-276.
[25] P.P. Roy, S. Paul, I. Mitra, K. Roy, On two novel parameters for validation of predictive QSAR models, Molecules 14 (2009) 1660-1701.

[26] B. Bhhatarai, P. Gramatica, Per-and polyfluoro toxicity (LC(50) inhalation) study in rat and mouse using QSAR modeling, Chemical Research in Toxicology 23 (2010) 528-539.

[27] H.Zhu, A. Tropsha, D. Fourches, A. Varnek, E. Papa, P. Gramatica, T. Oberg, P. Dao A. Cherkasov, I.V. Tetko, Combinatorial OSAR modeling of chemical toxicants tested against Tetrahymena pyriformis, Journal of Chemical Information and Modeling 48 (2008) 766-784.

[28] European Centre for Ecotoxicology and Toxicology of Chemicals, Aquatic toxicity data evaluation, Technical Report No. 56, Brussels, Belgium, 1993.

[29] S.J. Enoch, M.T.D. Cronin, T.W. Schultz, J.C. Madden, An evaluation of globa QSAR models for the prediction of the toxicity of phenols to Tetrahymena pyriformis, Chemosphere 71 (2008) 1225-1232.

[30] S. Spycher, P. Smejtek, T.I. Netzeva, B.I. Escher, Toward a class-independent quantitative structure-activity relationship model for uncouplers of oxidative phosphorylation, Chemical Research in Toxicology 21 (2008) 911-927.

[31] T.W. Schultz, Structure-toxicity relationships for benzenes evaluated with Tetrahymena pyriformis, Chemical Research in Toxicology 12 (1999) 1262-1267.

[32] V. Consonni, R. Todeschini, M. Pavan, Structure/response correlations and similarity/diversity analysis by GETAWAY descriptors. 1 . Theory of the novel 3D molecular descriptors, Journal of Chemical Information and Computer Science 42 (2002) 682-692.

[33] A.R. Katritzky, V.S. Lobanov, M. Karelson, QSPR: the correlation and quantitative prediction of chemical and physical properties from structure, Chemical Society Reviews 24 (1995) 279-287.

[34] A.G. Mercader, A.B. Pomilio, OSAR study of flavonoids and biflavonoids as influenza H1N1 virus neuraminidase inhibitors, European Journal of Medical Chemistry 45 (2010) 1724-1730.

[35] E.M. Boyd, K. Killham, A.A. Meharg, Toxicity of mono-, di- and tri-chlorophenols to lux marked terrestrial bacteria, Burkholderia species Rasc c2 and Pseudomonas fluorescens, Chemosphere 43 (2001) 157-166.

[36] A.O. Aptula, S.J. Enoch, D.W. Roberts, Chemical mechanisms for skin sensitization by aromatic compounds with hydroxy and amino groups, Chemical Research in Toxicology 22 (2009) 1541-1547.

[37] T. Satoh, S. Saitoh, M. Hosaka, K. Kosaka, Simple ortho- and para-hydroquinones as compounds neuroprotective against oxidative stress in a manner associated with specific transcriptional activation, Biochemical and Biophysical Research Communications 379 (2009) 537-541.

[38] J. Devillers, P. Boule, P. Vasseur, P. Prevot, R. Steiman, F. Seigle-Murandi, J.L. Benoit-Guyod, M. Nendza, C. Grioni, D. Dive, P. Chambon, Environmenta health risks of hydroquinone, Ecotoxicology and Environment Safety 19 (1990) 327-354.

[39] V.V. Lyakhovich, V.A. Vavilin, N.K. Zenkov, E.B. Menshchikova, Active defense under oxidative stress. The antioxidant responsive element, Biochemistry (Moscow) 71 (2006) 962-974. 